\title{
Surgical management of unruptured posterior carotid artery wall aneurysms
}

\author{
Brian A. O'Shaughnessy, M.D., Christopher C. Getch, M.D., \\ BernaRd R. BENDOK, M.D., AND H. Hunt BATJER, M.D.
}

Department of Neurological Surgery, The Feinberg School of Medicine and McGaw Medical Center, Northwestern University, Chicago, Illinois

\begin{abstract}
Intracranial aneurysms arising from the posterior wall of the supraclinoid carotid artery are extremely common lesions. The aneurysm dilation typically occurs in immediate proximity to the origin of the posterior communicating artery and, less commonly, the anterior choroidal artery (AChA). Because of the increasingly widespread use of noninvasive neuroimaging methods to evaluate patients believed to harbor cerebral lesions, many of these carotid artery aneurysms are now documented in their unruptured state, prior to occurrence of subarachnoid hemorrhage. Based on these factors, the management of unruptured posterior carotid artery (PCA) wall aneurysms is an important element of any neurosurgical practice.

Despite impressive recent advances in endovascular therapy, the placement of microsurgical clips to exclude aneurysms with preservation of all afferent and efferent vasculature remains the most efficacious and durable therapy. To date, an optimal outcome is only achieved when the neurosurgeon is able to combine systematic preoperative neurovascular assessment with meticulous operative technique. In this report, the authors review their surgical approach to PCA wall aneurysms, which is greatly based on the extensive neurovascular experience of the senior author. Focus is placed on their methods of preoperative evaluation and operative technique, with emphasis on neurovascular anatomy and the significance of oculomotor nerve compression. They conclude by discussing surgery-related complications, with a particular focus on intraoperative rupture of aneurysms and their management, and the postoperative ischemic AChA syndrome.
\end{abstract}

\section{KEY WORDS • carotid artery - intracranial aneurysm • unruptured aneurysm • posterior communicating artery $\bullet$ anterior choroidal artery}

Intracranial aneurysms arising from the supraclinoid carotid artery in proximity to the origin of the PCoA or AChA, together referred to as PCA wall aneurysms, are common lesions in vascular neurosurgery. More specifically, PCA wall aneurysms account for approximately $30 \%$ of all cerebral aneurysms and greater than $50 \%$ of those affecting the CA. Considered separately, PCoA aneurysms are much more common than AChA aneurysms, the latter constituting at most $5 \%$ of all intracranial aneurysms. ${ }^{7,16,25}$ Because of their high relative frequency, these lesions require skillful surgical management in any neurovascular practice. Despite their frequency, however, their treatment is paradoxical: although they are rather easily exposed, placement of clips without complications can be quite difficult because of critical surrounding neurovascular structures. One must never approach PCA wall aneurysms with complacency; even the most mundane intracranial vascular disease should be treated with utmost care. The senior author (H.H.B.) heard the late Dr. Charles

Abbreviations used in this paper: $\mathrm{AChA}=$ anterior choroidal; $\mathrm{AP}=$ anteroposterior; artery; $\mathrm{CA}=$ carotid artery; $\mathrm{CSF}=$ cerebrospinal fluid; $\mathrm{PCA}=$ posterior $\mathrm{CA}$; $\mathrm{PCoA}=$ posterior communicating artery.
Drake state many times that "posterior communicators are either the simplest or the most disastrous aneurysms to treat."

Despite the technological advances in endovascular coil therapy, microsurgical clip exclusion remains the most definitive and time-honored management strategy for cerebral aneurysms if it can be undertaken safely. An immediate and long-lasting cure, an angiographically verifiable result, and complete mechanical uncoupling of the aneurysm tissue from the intracranial circulation are provided. In this report, we discuss our methods for evaluating and surgically treating PCA wall aneurysms, which are largely based on the extensive neurovascular experience of the senior author (H.H.B.) in the surgical management of ruptured and unruptured lesions. Also discussed will be intraand postoperative complications, the latter of which have only been recently well-characterized by the Mayo Clinic group in their appraisal of ischemic AChA insults.

\section{UNRUPTURED PCA WALL ANEURYSMS}

\section{Aneurysm Pathogenesis}

The propensity for aneurysms to originate from the posterior wall of the supraclinoid CA is undoubtedly related 
to the unique anatomy and arterial flow dynamics in this region of the cerebral vasculature. Rhoton, alone ${ }^{18-20}$ and with colleagues, ${ }^{22}$ in his role as a microsurgical neuroanatomist, is credited with introducing four anatomical axioms directly applicable to the development and treatment of intracranial saccular aneurysms. His principles, while described in terms of anatomical relationships, have clearly been derived from the physiological nature of fluid flow under pressure, underscoring the structure-function interplay that is ubiquitous in biological systems.

Dilation of a vessel wall and generation of an aneurysm results from hemodynamic stress over time, particularly at points of vascular tissue vulnerability, such as sites of turbulent blood flow that can be imposed by vessel tortuosity. The PCA wall, which possesses a sharply demarcated posteriorly oriented convexity, is both a site of turbulent blood flow and significant hemodynamic stress as it carries considerable cerebral inflow. This region is therefore at significant risk for development of aneurysms. The apex of this convexity, the location of maximal transmural pressure, is at greatest risk of aneurysm formation. Usually, the PCoA arises from the CA closest to the posterior convexity apex and in these patients a PCoA aneurysm results. By contrast, it would follow that AChA aneurysms are more prone to develop in patients in whom the vascular anatomy is such that the AChA arises closest to the apex. Whether the aneurysm lies near the origin of the PCoA or the AChA appears to be, based on Rhoton's axioms, arbitrarily dictated by the particular PCA anatomy. Nevertheless, such a distinction carries important surgical significance: operative risks are usually far greater in the case of AChA aneurysms due largely to the greater spectrum of critical choroidal segment perforators and the AChA itself, an unequivocally important vessel with which one must successfully negotiate for optimal clip application and parent artery reconstruction.

\section{Preoperative Considerations}

Oculomotor Nerve Compression. The most common structure compromised by a PCA wall aneurysm, particularly characteristic of a posterolaterally expanding PCoA aneurysm when it reaches 4 to $5 \mathrm{~mm}$ in maximal diameter, ${ }^{19}$ is the oculomotor nerve (Fig. 1). Clinically, intracranial aneurysms producing mass effect have a significantly increased incidence of rupture (estimated annual risk $6 \%{ }^{24}$ ). It has been suggested that aneurysm-induced oculomotor nerve palsy results from localized mechanically mediated nerve compression or from hemorrhagic dissection of the nerve as it exits the mesencephalon and courses in the interpeduncular fossa toward the orbit. ${ }^{11}$ Because mechanical compression is the most likely mechanism of aneurysm-induced nerve palsy in the large majority of cases, dysfunction of the parasympathetic fibers is more common than paralysis of the somatic motor components, although frequently both are affected. In very rare instances, a PCoA aneurysm may actually fenestrate the oculomotor nerve, a condition that may necessitate sacrificing portions of the nerve to facilitate complete surgical obliteration of the aneurysm. ${ }^{11,25}$

Patients presenting with a pupillary-sparing oculomotor nerve palsy pose a unique challenge because transient third nerve palsy may occur in patients with diabetes mel- litus, myasthenia gravis, or inflammatory conditions. Oculomotor nerve palsy in association with the aforementioned conditions most commonly affects somatic motor function of the nerve; it is thought to result from microvascular ischemia to the vasa nervorum in diabetes and to compromise the cholingeric neuromuscular junction in myasthenia gravis. Frequently, these conditions spare the peripherally located parasympathetic fibers, and hence, the pupil is usually reactive to light. By contrast, it is very uncommon for oculomotor nerve palsy resuting from an aneurysm to spare the pupil. Nevertheless, cases of pupillary-sparing oculomotor palsies resulting from PCoA aneurysms have been reported. ${ }^{13}$ One helpful feature in the differentiation of aneurysm-induced oculomotor paresis is the presence of retroorbital pain, a commonly described symptom in patients with an expanding PCoA aneurysm. It is our belief, however, that a patient with an oculomotor nerve palsy of any type, particularly if it induces pain, should be considered to harbor a PCA wall aneurysm until proven otherwise. Diagnostic investigation can begin with computerized tomography angiography in such cases.

Clinical data exist that suggest that the timing of aneurysm surgery in relation to the development of aneurysminduced oculomotor nerve compression is related to the ultimate recovery of nerve function. ${ }^{10,15}$ Early surgery (within the 1st week after onset of oculomotor palsy) is associated with an excellent chance of nerve function recovery. By contrast, late surgery ( $>4$ weeks after onset of oculomotor palsy) is associated with a poor chance of nerve function recovery. In their small clinical series, Leivo and colleagues ${ }^{15}$ found that of the nine patients who underwent surgery within 3 days of experiencing aneurysm-induced oculomotor palsy full recovery of nerve function occurred in eight (88.9\%); of the six patients in whom surgery was performed between 4 and 6 days complete recovery of nerve function occurred in only four (66.7\%); and of those six patients in whom surgery was performed on more than 1 month after aneurysm-induced nerve palsy, only complete return of oculomotor nerve function occurred in one (16.7\%). Furthermore, it has been suggested that when oculomotor function recovery begins within the 1st postoperative month, recovery will probably eventually be complete, as is demonstrated in cases of neurapraxia from conduction block or minor axonal disturbance. ${ }^{10}$ Based on this evidence, patients with aneurysm-induced oculomotor nerve compression should be immediately admitted to the hospital, preferably within the first 3 days of onset, and prompt operative treatment of the aneurysm with neural decompression should be provided. Such an approach is undertaken not only to ensure the timely recovery of oculomotor nerve function but also to avoid subarachnoid hemorrhage, because the onset of palsy is thought to signify an unstable lesion with the threat of impending rupture.

Vascular Anatomy of the PCoA. The PCoA, which forms the lateral boundary of the circle of Willis, originates from the posteromedial surface of the supraclinoid CA and courses approximately 5 to $10 \mathrm{~mm}$ posteromedially above the sella turcica to anastomose with the PCA at the $\mathrm{P}_{1}-\mathrm{P}_{2}$ junction. During fetal development, the PCoA continues as the PCA; however, in adults, the principal arterial flow to the PCA territory is assumed by the vertebrobasilar artery system. As such, in the majority of pa- 


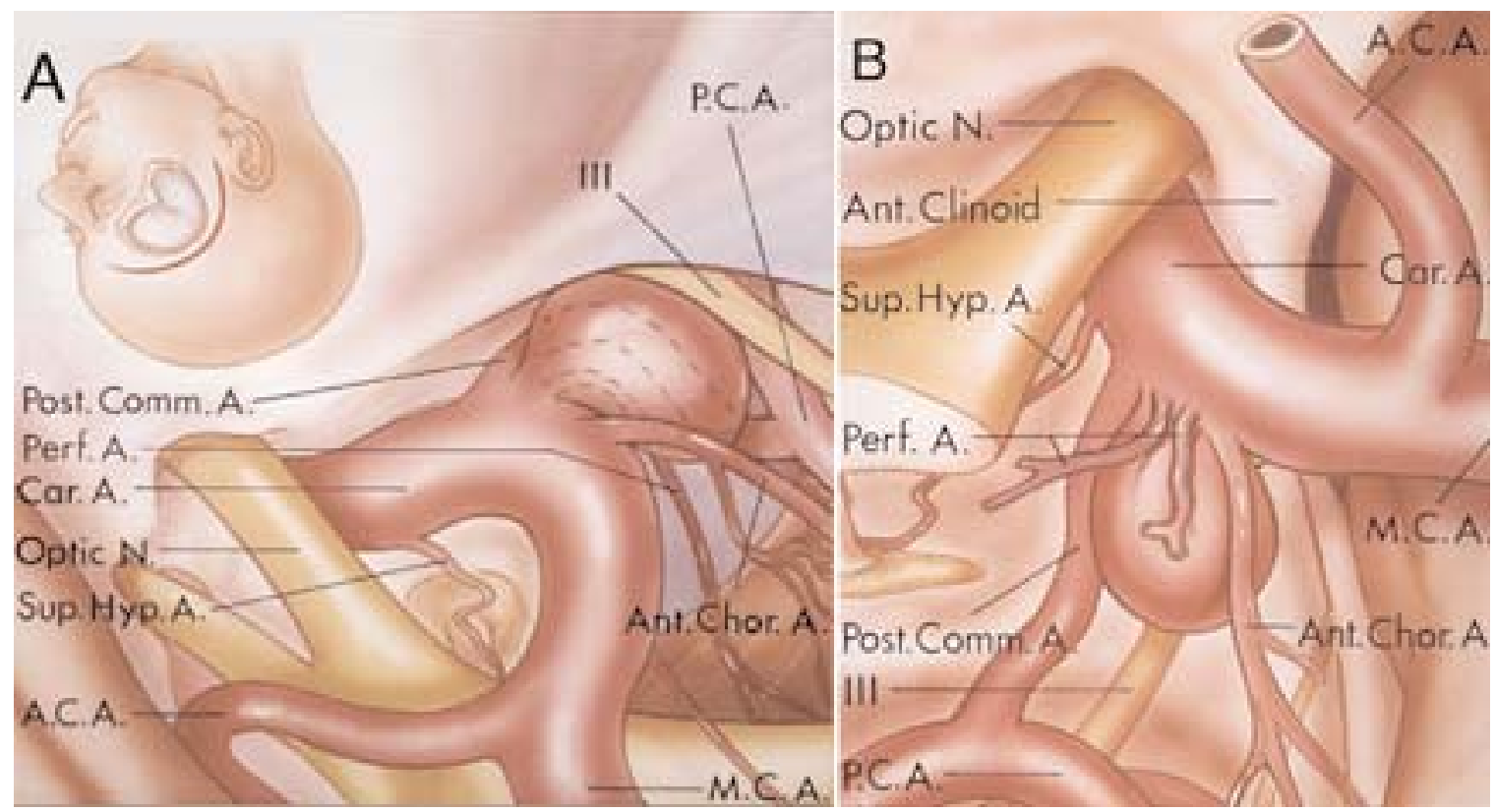

Fig. 1. Illustration depicting lateral (A) and superior (B) operative views of a saccular aneurysm arising from the CA at the distal edge of the origin of the PCoA (Post Comm A). The aneurysm clearly projects posterolaterally and compresses the oculomotor nerve. Another important feature to note is that the main trunk of the AChA is juxtaposed and adherent to the sac of the aneurysm. This is critical because meticulous preservation of the integrity of this vessel is of vital significance during both microdissection and clip application. $\mathrm{A}=$ artery; ACA = anterior cerebral artery; Ant Chor = AChA; MCA = middle cerebral artery; Perf = perforating; Sup Hyp = superior hypophysial; III = third cranial nerve. Reproduced with permission from Rhoton Jr: Aneurysms. Neurosurgery 51 Suppl 1:121-158, 2002.

tients, the PCoA is patent but smaller caliber than the ipsilateral PCA with which it anastomoses. For this reason, the functional role of the PCoA is typically to serve as an anastomotic bridge between the anterior and posterior circulations, as well as to supply anterior thalamoperforatoring vessels.

In an estimated $20 \%$ of the population, the PCoA is found to be larger than the ipsilateral $\mathrm{P}_{1}$ segment, and in these cases the PCoA remains the principal origin of the PCA. When the PCoA is the sole arterial supply of the PCA territory, the vascular configuration is termed "fetal" and the patient is said to harbor a fetal PCA (Figs. 2 and 3 ). Because of the vascular territory that it subserves, a fetal PCA is often found superolateral to the oculomotor nerve instead of the typical superomedial location present in patients with the common "adult" circulation. The presence of a fetal PCA ipsilateral to a PCA wall aneurysm is important because it has significant implications during surgery. Occlusion of a PCoA in a patient with a patent and normal caliber $\mathrm{P}_{1}$ segment is usually tolerated quite well. By contrast, inadvertent sacrifice of a fetal PCA may result in complete territorial infarction depending on the level at which the occlusion occurs, as well as preexisiting collateralization. Such an anatomical configuration warrants far more intraoperative care than is usually given to a PCoA because, in a fetal pattern of circulation, this vessel is not just an anastomotic channel but rather a major source of vascular inflow to critical neural structures.

Aneurysm Projection and Neck Anatomy. Preoperatively it can be highly valuable to determine the projection of a PCA wall aneurysm, which frequently requires multi- ple angiographic views including lateral, AP, and oblique projections. Computerized tomography angiography can now demonstrate exquisite three-dimensional anatomical detail of this area, which often greatly aids in preoperative planning. Posterior communicating artery aneurysms are variable with respect to their projection; however, a posterolateral projection with potential compression of the oculomotor nerve is most common and is present in approximately $85 \%$ of cases. Frequently, PCoA aneurysms with this projection overlie the $\mathrm{PCoA}$ and its origin from the CA is therefore obscured. By contrast, PCoA aneurysms that project superolaterally often spare the oculomotor nerve and must be assumed to lie above the tentorial edge and be tightly adherent to the medial aspect of the temporal lobe. Rupture of such an aneurysm may be associated with concomitant temporal lobe hemorrhage in addition to hemorrhage into the subarachnoid space.

In cases of superolaterally projecting PCoA aneurysms that spare the oculomotor nerve, the surgeon must only retract the frontal lobe for the initial dissection and absolutely refrain from temporal lobe retraction to avoid causing iatrogenic aneurysm rupture. By contrast, when the aneurysm projects posterolaterally in its usual fashion, it lies below the tentorium and is not adherent to the temporal lobe; thus, gentle temporal retraction is permissible. In this case, the presence or absence of an oculomotor palsy can have considerable implications for surgery, which underscores the value of clinicoanatomical localizationby preoperative neurological examination and detailed neuroimaging - in guiding the approach intraoperatively. This being stated, however, because the aforementioned 

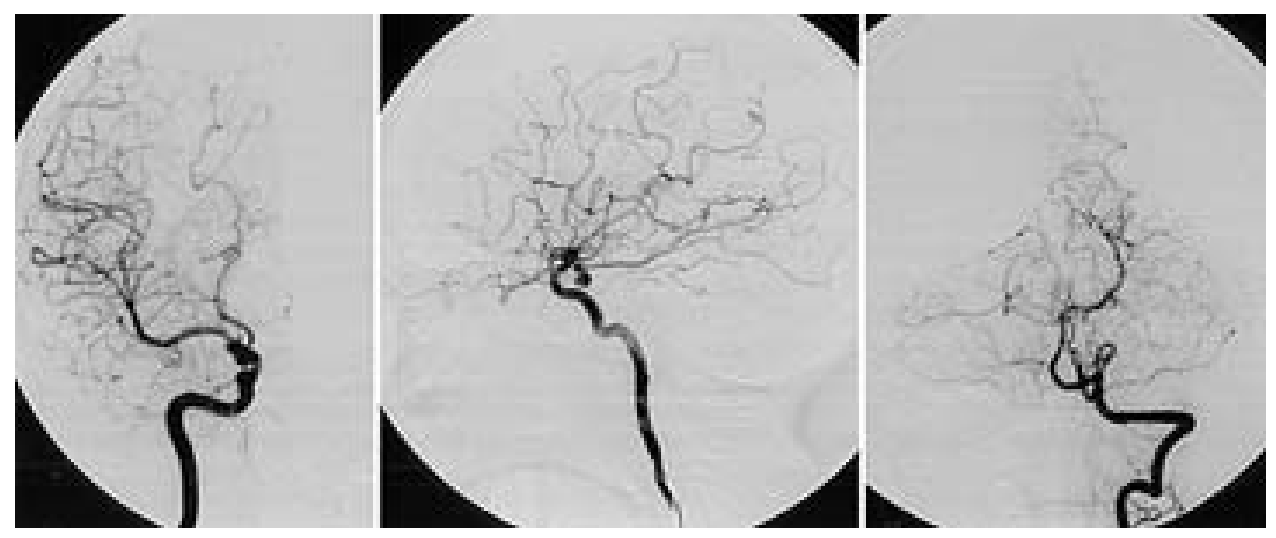

Fig. 2. Left and Center: Left CA AP (left) and lateral (center) digital subtraction angiograms. A multilobulated aneurysm, approximately $10 \times 15 \mathrm{~mm}$ in diameter, can be seen arising from the CA at the origin of the PCoA. Of note, the morphological irregularity of the lesion suggests recent rupture. Right: Left vertebral AP angiogram demonstrating a right fetal PCA, an anatomical variant with surgical significance when ipsilateral to a PCA wall aneurysm, as in this case. Unlike a typical PCoA, which can often be surgically sacrificed without undue consequences, loss of a fetal PCA in this patient could result in a major territorial infarction. This angiographic finding underscores the importance of obtaining a complete four-vessel cerebral angiographic study prior to operative treatment of any PCA wall aneurysm.

generalizations regarding aneurysm projection are clearly not without exception, any retraction of the temporal lobe should be performed using extreme caution; additionally, it is most prudent to reserve this retraction until the very final stage of microdissection if possible, during neck exposure and clip application.

In contrast to PCoA aneurysms, AChA aneurysms often arise from the inferior surface of the CA, project inferolaterally, and almost always, therefore, spare the oculomotor nerve. Of note, AChA aneurysms may possess a broad base and, for this reason, assume a configuration not readily amenable to conventional surgical clip application. In such cases, a prolonged period of temporary occlusion may be required to allow for both aneurysm clipping and parent artery reconstruction; it should be noted, however, this occurrence is quite rare.

Timing of Surgery. One may broadly divide any discussion on the timing of aneurysm surgery into the fol-

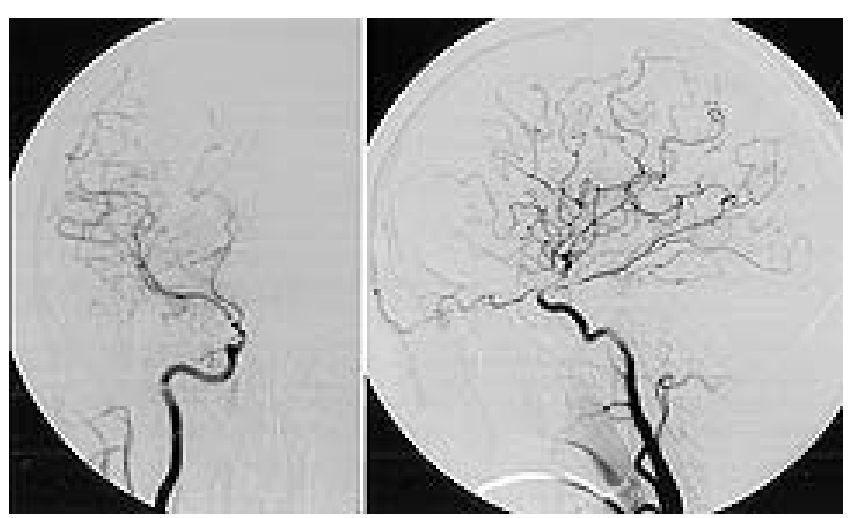

Fig. 3. Postoperative AP (left) and lateral (right) cerebral angiograms obtained in the same patient represented by images in Fig. 2. Complete uncoupling of the aneurysm from the intracranial circulation can be seen, as can widely patent flow through both the fetal PCA and CA. lowing categories: ruptured lesions, unruptured but symptomatic lesions, and incidental lesions. In cases in which the aneurysm is unruptured, many factors including aneurysm size, irregularity of size on angiography, patient age, and clinical symptoms must be taken into account when choosing the management strategy and timing of the intervention. As previously stated, aneurysm obliteration achieved by the application of clips is the most definitively curative treatment, and in patients who can withstand an operation, it is the intervention of choice. It is our opinion that characteristics such as young age, presence of clinical symptoms unrelated to rupture such as mass effect, aneurysm irregularity with the presence of daughter sacs, and size larger than $7 \mathrm{~mm}$ all indicate the need for treatment; however, treatment must be tailored to the individual, taking not only each of these factors into account but also the impact of an unruptured intracranial aneurysm on a patient's life.

\section{Operative Technique}

Posterior CA wall aneurysms are approached via an ipsilateral frontotemporal craniotomy in which the cranial bone flap is centered at the pterion. The standard approach may be modified when more than one aneurysm of the anterior circulation or a concomitant vertebrobasilar lesion is present. Antistaphylococcal agents and dexamethasone are both routinely administered prior to the start of the procedure. Electroencephalographic leads are placed should burst suppression and temporary arterial occlusion be required during clip application and CA reconstruction. Coincident with the scalp incision, osmotic diuresis with mannitol is conducted to relax the brain and allow it to be gravitationally displaced. The head of the patient should, in general, be positioned according to the projection of the aneurysm to allow gravitational assistance in brain retraction; this requires the patient to be supine and the head rotated approximately $30^{\circ}$ to the contralateral side with the malar eminence at the apex. Further rotation in the contralateral direction causes the mesial temporal lobe to encroach upon the incisura, a clearly undesired event. 
The skin incision is started at the level of the zygomatic arch, approximately $1 \mathrm{~cm}$ anterior to the tragus, and gently curved anteriorly until it reaches the midline at the hairline -forehead interface. A scalp flap is elevated above the level of the temporalis muscle and held under mild traction with the aid of fish hooks. A relaxing incision is made in the superficial temporalis fascia to lessen the chance of inducing traction injury to the frontal branch of the facial nerve supplying the forehead (interfascial dissection). A free pterional-centered bone flap is then generated with the use of a high-speed pneumatic drill. The lateral aspect of the sphenoid ridge is judiciously resected using both the pneumatic drill and rongeurs to create an unobstructed view of the anterior clinoid process. With the systemic blood pressure maintained in the normotensive range, the dura mater is then carefully opened in a semicircular fashion and secured over the submuscular scalp flap to tamponade any epidural bleeding. At this stage, further brain relaxation, if necessary, is easily achieved using the Paine technique ${ }^{17}$ to insert a ventriculostomy.

Once the dura is opened and the ventricular system is adequately decompressed, a microscope is brought into the operative field and microsurgical dissection is commenced. Throughout the dissection, the neurosurgeon must refrain from excessive brain retraction. Proximal vascular control of the CA should be established early to prepare for intraoperative rupture should it occur. The procedure is focused on rigorous dissection and identification of all vital neurovascular structures prior to initiating clip application. Strict avoidance of blunt dissection is critical; one must use sharp techniques throughout the operation, which is especially important during dissection of the aneurysm neck if rupture is to be avoided.

The initial microdissection is directed toward the chiasmatic cistern overlying the ipsilateral optic nerve lying just medial to the CA. One uses the microknife and microscissors carefully to divide the arachnoidal adhesions bridging the optic nerve to the undersurface of the frontal lobe, thus allowing gentle frontal lobe retraction without undue traction on the optic apparatus. When the optic nerve is liberated from its overlying cerebral adhesions, a self-retaining brain retractor is introduced into the operative field and the frontal lobe gently retracted to create an adequate aperture through which the operation may proceed. Again using the microknife and microscissors, the arachnoid is opened into the CA cistern and carried along the medial aspect of the CA to the level of the anterior clinoid process. Proximal vascular control is usually easily established just above the site at which the CA pierces the dural roof of the cavernous sinus. Gentle CSF drainage through this opening will provide additional working space. In rare cases involving low-lying PCA wall aneurysms or an unusually prominent anterior clinoid process, opening of the distal dural ring and even resection of the anterior clinoid process may be necessary to achieve adequate exposure of the aneurysm neck.

After proximal control is established, the microdissection then proceeds distally along the medial border of the CA to obtain distal vascular control just proximal to the CA bifurcation. The medial portion of the sphenoidal sylvian fissure must be divided to visualize the distal $\mathrm{CA}$ without requiring excessive brain retraction. Precise identification of the course of the AChA is not readily achieved without some sylvian fissure dissection. It is critical to spend as much time as needed identifying and sharply dissecting the AChA and any perforating vessels arising from the communicating or choroidal segments. Dissection is typically undertaken in a distal-to-proximal fashion along the lateral wall of the $\mathrm{CA}$ to permit precise identification of the origin of the AChA.

In cases in which temporary clip application is anticipated to allow for permanent clip occlusion and CA reconstruction, the dissection should be extended proximal to the AChA to exclude this critical vessel from the temporarily occluded segment. When a PCoA aneurysm is being treated, the AChA is frequently adherent to the fundus of the aneurysm, and great care should be taken to free this adhesion. If a clip closes the aneurysm and the AChA remains adherent, an accordion effect can compromise the lumen of this fragile vessel by kinking its wall; this might lead to a devastating postoperative infarction. The PCOA must be identified not only at its origin from the CA posterolaterally, but also medially through the opticocarotid triangle, which is an important step if the PCoA is of large caliber or if the circulation has a fetal configuration. It may actually be necessary to manipulate the CA to identify and allow the PCoA to be dissected, particularly in cases where its initial visualization is obscured. In doing so, one should refrain from any medial retraction of the CA toward the optic nerve, because this maneuver may cause rupture of the PCoA aneurysm dome when counter traction is exerted by the surrounding neural elements, such as the temporal lobe, petroclinoid ligament, or tentorium, to which it is attached.

Only after all critical neurovascular structures are dissected and vascular control is strictly established can the neurosurgeon proceed safely with dissection of the aneurysm neck under high microscopic magnification. The aneurysm neck should be dissected before the fundus because the former aspect can tolerate the greatest manipulation, is the least likely portion to rupture, and is the ultimate location where the permanent clip will be placed. Careful microdissection of arterial branches arising near the aneurysm neck is crucial prior to clip application to avoid inadvertent incorporation of a critical vessel inside the clip. The separation of small arterial branches and perforating vessels from the aneurysm may be facilitated by temporary clip occlusion of the CA to reduce the transmural pressure within the aneurysm, thereby allowing relatively free manipulation of the sac. Temporary clip occlusion should be conducted using pharmacologically induced burst suppression and moderate hypothermia (range $32-33^{\circ} \mathrm{C}$ ) to ensure adequate ischemic protection.

Following careful microdissection of the aneurysm neck, clip application under strict direct visualization is carefully performed. A straight clip that is just long enough to occlude the entire neck is usually optimal. A curved clip may result in the inadvertent incorporation of perforators, the AChA, or PCoA within the clip because their distal course is hidden behind the CA. By contrast, an excessively long clip can incorporate the oculomotor nerve. It should be remembered that when estimating the length of clip required for complete neck occlusion, the neck becomes approximately $33 \%$ wider than its initial 
dimension when compressed. After clip application, rigorous visual scrutiny is necessary to ensure that no arterial branch, perforating vessel, or the oculomotor nerve is inadvertently incorporated between the clip blades. When the clip is satisfactorily positioned and no pulsations are noted in the aneurysm, the dome is punctured.

Although we do not routinely utilize intraoperative angiography, it is useful in the setting of a complex PCoA aneurysm with the presence of a fetal PCA as well as in cases involving AChA aneurysms with clearly threatening configurations or those which are giant in size. Postoperative angiography should be routinely performed until the neurosurgeon is able to judge in which cases selective postoperative angiography would be best suited to assess clip exclusion of the aneurysm and parent vessel patency. Prior to the replacement of the cranial bone flap, a watertight dural closure is obtained to prevent CSF leakage. Soft acrylic may be molded into both the cranial burr holes and sphenoid wing defect to minimize the already minimal cosmetic impact of the pterional approach.

\section{Intraoperative Aneurysm Rupture}

Because intraoperative rupture should be anticipated at every stage of the procedure, the team should be armed with an evolving plan of defense. It has been suggested that few other surgical situations exist for which the outcome is so largely contingent on the skills of the surgeon than intraoperative aneurysm rupture; it can be either a brief disturbance or a significant impedance to successful vessel reconstruction. ${ }^{1-3,14,23,65}$ Much of our modern understanding of intraoperative aneurysm rupture, from a mechanistic and management perspective, has resulted from the work by Batjer and Samson. ${ }^{3}$ In excellent hands, the incidence of intraoperative rupture is estimated to be approximately $8.5 \%{ }^{36}$ Rupture may occur at any stage of the procedure, but in our experience and that of others, ${ }^{1,9,14,23,65}$ it most commonly occurs during the final approach and microdissection of the aneurysm neck prior to clip placement. General techniques to avoid intraoperative rupture involve adherence to standard principles of sharp microdissection, careful hemodynamic monitoring to avoid hypertension, and minimization of brain retraction by using pharmacologically induced brain relaxation and ventricular or spinal catheterization.

Once general principles are taken into account, each specific aneurysm has unique features, referable to the regional anatomy that can be used to plan operative strategies to avoid intraoperative rupture. ${ }^{14,23}$ Aneurysms of the PCA wall are known, for several reasons, to be associated with a higher incidence of intraoperative rupture than aneurysms in other locations. Rupture during surgery of PCA wall aneurysms can be divided into three categories based on the time at which it occurs: 1) during temporal lobe retraction; 2) when advancing the clip blades; and 3) at the time of clip closure.

As previously stated, PCA wall aneurysms, particularly laterally projecting PCoA aneurysms, tend to be adherent to the medial aspect of the temporal lobe, the dome of which usually forms a bridge between the relatively immobile CA and readily movable temporal lobe. As such, intradissection movement of the temporal lobe can predispose to rupture. For this reason, every effort should be made to reserve temporal lobe retraction for as late in the procedure as possible, prior to clip application. When neurological examination demonstrates an oculomotor nerve palsy, its identification can help in the manner in which temporal lobe retraction is conducted intraoperatively.

If rupture occurs during clip blade advancement, the blades should not be advanced further because the distal clip blades have likely punctured the aneurysm sac. The clip should be immediately withdrawn and temporary clip occlusion of the CA proximal to the origin of the vessel with which the aneurysm is associated should be performed to reduce the amount of blood in the operative field and to restore anatomical visualization. Temporary clips should be applied proximally, distally, and on the PCoA itself in that order until the field is clear enough to continue working. If complete trapping of the aneurysm is necessary, the neurosurgeon must keep in mind the duration of the occlusion, particularly if the choroidal segment is included in the trapped region. After CA control has been established, the neurosurgeon must perform expedient and meticulous dissection of the aneurysm neck through the opticocarotid triangle. Revisualization of the exact location of the AChA and its relationship to the aneurysm neck is crucial prior to permanent clip application to avoid inadvertent vessel injury.

The final mechanism responsible for intraoperative rupture of PCA wall aneurysms occurs during clip blade closure. This occurs because the aneurysm is frequently adherent to neighboring neurovascular structures including the medial temporal lobe or tentorial incisura. When the clip is applied across the neck and the blades are approximated, the aneurysm-neurovascular structure interface is placed under greater pressure than the aneurysm wall can withstand, resulting in a tear and subsequent hemorrhage. In other words, intraoperative rupture may occur when the fundus is "gathered" during clip blade closure and it is avulsed from the tentorium. In this situation, if the aneurysm neck has been well dissected and secured by the clip blades, complete closure will almost certainly abate the hemorrhage.

\section{Postoperative Complications}

Cranial Nerve Injury. Unintentional injury to one of the upper cranial nerves is possible during surgery for a PCA wall aneurysm and can be considered, depending on the injury, a significant complication. During the initial stage of a standard pterional craniotomy, damage to the olfactory tract can occur and is frequently associated with initial elevation of the frontal lobe to expose the CA, retraction of the frontal lobe, or bipolar cautery of small bleeding veins in the region of the tract. These problems are initially avoided by generous CSF decompression and gentle retraction of the frontal lobe by careful manipulation with the aid of a brain retractor. We recommend placing adaptic gauze on the undersurface of the frontal lobe over the olfactory tracts prior to the introduction of brain retractors into the field. Judicious usage of gelfoam to control bleeding in the region of the cribriform plate is also a valuable adjunctive measure.

Injury to the optic apparatus or oculomotor nerve- the other two cranial nerves at risk-may occur during both exposure and manipulation of the aneurysm. In addition, 
the oculomotor nerve is susceptible to iatrogenic injury during clip application when it is inadvertently incorporated between the clip blades. These complications are avoided using sharp microdissection techniques, vigilant attention to all neurological structures in the operative field, and careful mobilization of the aneurysm during final clip application.

Fetal PCA Injury. As stated previously, a fetal PCA is a relatively common anatomical variant in which the PCoA provides arterial supply through the anterior circulation to the PCA territory. As such, aneurysms of the supraclinoid CA at the origin of a fetal PCA are fraught with potential danger. As described earlier, inadvertent sacrifice of a PCoA is usually tolerated quite well; however, interruption of arterial supply through a fetal PCA can result in massive infarction of the midbrain, thalamus, and ipsilateral cerebral hemisphere, a truly devastating and irreconcilable event. In this sense, a fetal PCA, if it exists, must be protected during any CA aneurysm surgery. In fact, we believe its presence is a strong indicator that intraoperative angiography should be performed after clip placement to ensure patent blood flow through the vessel.

Injury to the AChA. In an early report by Drake and colleagues $^{6}$ surgical treatment of AChA aneurysms was associated with profound complications when the blood flow through the AChA was interrupted. In their early report, they stated that when surgically treating AChA aneurysms, the "intimate relationship to the choroidal artery has surgical significance, for inadvertent injury to this vessel may have disastrous complications." We and many others readily agree with this statement. Such an infarction typically results in hemiplegia, hemianesthesia, and hemianopsia, ${ }^{4,5,12}$ although variability in the clinical syndrome certainly exists.

The most recent, and certainly most comprehensive, account of this complication has been described by Fried- man and coworkers ${ }^{8}$ who reported a 28 -year experience at the Mayo Clinic in the surgical treatment of $51 \mathrm{AChA}$ aneurysms in 50 patients. Of the 50 patients undergoing surgery for AChA aneurysms, in eight patients clinical and radiographic evidence of AChA ischemia was consistent with infarction of the posterior limb of the internal capsule, the most poorly collateralized region supplied by the AChA. ${ }^{21}$ Hemiplegia was present in all eight patients, whereas hemisensory loss and hemianopsia were documented in one and two patients, respectively. This inconsistency of clinical findings with respect to hemisensory deficits and visual field cuts is suggestive of considerable individual variation with respect to diencephalic and optic apparatus collateralization.

These data reported by Friedman and coworkers ${ }^{8}$ suggest that a postoperative AChA syndrome is not related to aneurysm rupture or the size at the time of rupture, but rather its specific configuration and its relationship to the AChA. Of the five anatomical variants of AChA aneurysms, two have been most consistently associated with a postoperative AChA syndrome. One high risk configuration is seen when the aneurysm originates from the main AChA trunk (Fig. 4 left); the other is observed when the AChA originates from the base of a wide-necked AChA aneurysm (Fig. 4 right). Both of these complex configurations make extremely challenging the direct clip application without interruption of blood supply through the AChA. In fact, Friedman and coworkers described purposeful sacrifice of the AChA in one of the eight patients with a postoperative AChA syndrome because the aneurysm neck had avulsed from the AChA itself, making simultaneous aneurysm clip occlusion and preservation of the vessel essentially impossible by conventional operative techniques. This patient awoke from surgery with a pure motor AChA syndrome for which no diagnostic or therapeutic intervention was subsequently performed.

There are no clear-cut solutions in the management of this dreaded complication. It is our belief that the first

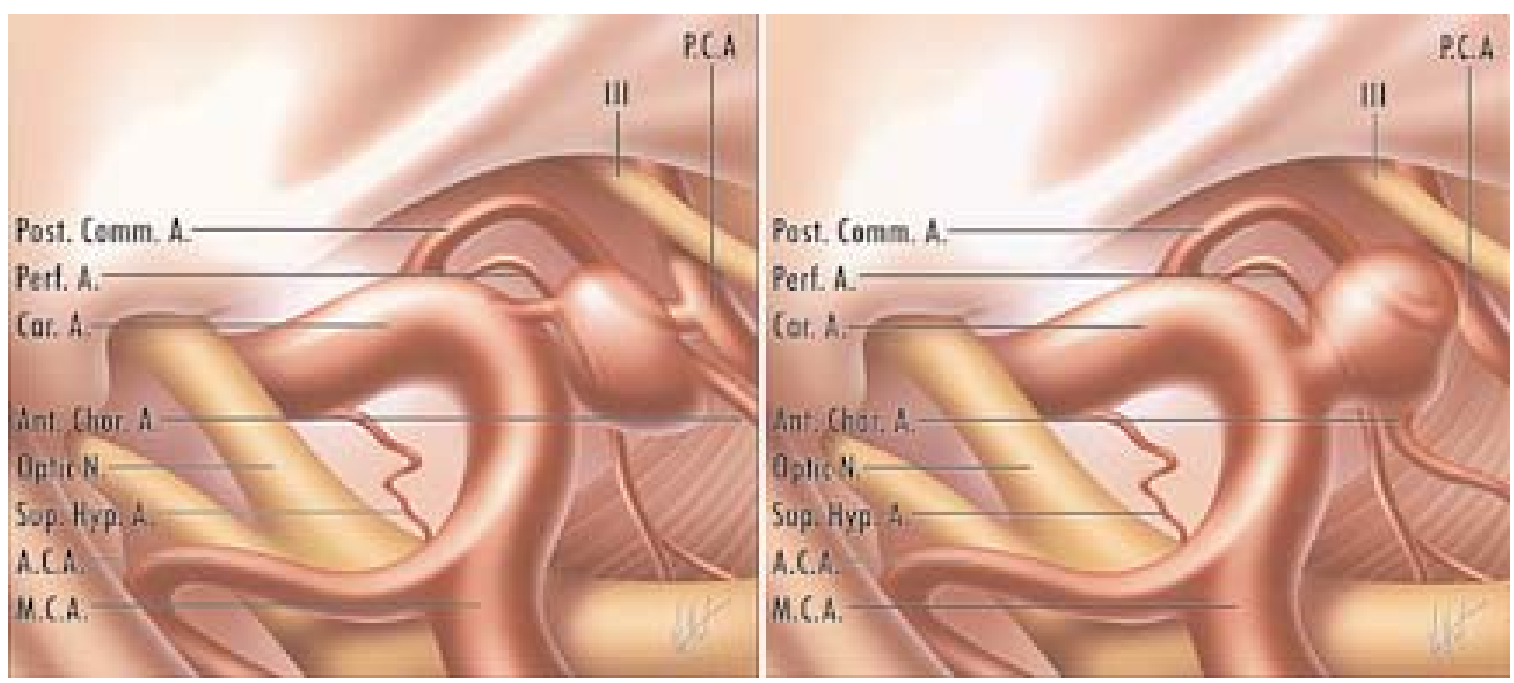

Fig. 4. Illustrations demonstrating the two principal configurations that predispose to the development of a postoperative ischemic ACA syndrome. Left: In one case, the aneurysm originates from the main AChA trunk. Right: In the other case, the AChA originates from the base of a wide-necked AChA aneurysm. Car A = CA. 
measure of treatment involves leaving a generous origin of the AChA. Moreover, the selective usage of intraoperative angiography and transcranial Doppler ultrasonography to demonstrate AChA patency and estimate AChA blood flow, respectively, should be used when the aneurysm appears to have a threatening configuration. In the event that an AChA syndrome develops postoperatively, despite a patent AChA at the time of closure, the neurovascular team should immediately return to the operating room for assessment of clip placement and its possible repositioning. After the aneurysm is secured, the patient should additionally receive hyperdynamic therapy to maximize cerebral perfusion, a treatment potentially beneficial in all patients after aneurysm surgery, even those without clinical or radiographic evidence of cerebral ischemia.

\section{CONCLUSIONS}

Posterior CA wall aneurysms, commonly encountered in any vascular neurosurgery practice, can be typically cured. They must be approached with deference, however, because they are associated with risks that can be quite significant. Close attention to the results of preoperative neurological assessment may help guide the operative maneuvers. Particular attention to the specific vascular anatomy and projection of the aneurysm demonstrated on preoperative angiograms can be of profound importance, particularly if a fetal PCA is present. Indeed, it is helpful to have angiograms from an AP vertebral injection hanging within the operating room next to AP and lateral ipsilateral CA injections just to remind the surgeon of the status of the $\mathrm{P}_{1}$ segment of the ipsilateral PCA. Successful surgery and avoidance of complications require a high level of operative skill as well as careful and detailed preoperative planning. All important neurovascular structures must be prudently and completely dissected, reserving clip application as the final stage after thorough microdissection.

\section{References}

1. Batjer H, Samson D: Intraoperative aneurysmal rupture: incidence, outcome, and suggestions for surgical management. Neurosurgery 18:701-707, 1986

2. Batjer HH, Frankfurt AI, Purdy PD, et al: Use of etomidate, temporary arterial occlusion, and intraoperative angiography in surgical treatment of large and giant cerebral aneurysms. J Neurosurg 68:234-240, 1988

3. Batjer HH, Samson DS: Causes of morbidity and mortality from surgery of aneurysms of the distal basilar artery. Neurosurgery 25:904-916, 1989

4. Bruno A, Graff-Radford NR, Biller J, et al: Anterior choroidal artery territory infarction: a small vessel disease. Stroke 20: 616-619, 1989

5. Decroix JP, Graveleau P, Masson M, et al: Infarction in the territory of the anterior choroidal artery. A clinical and computerized tomographic study of 16 cases. Brain 109:1071-1085, 1986
6. Drake CG, Vanderlinden RG, Amacher AL: Carotid-choroidal aneurysms. J Neurosurg 29:32-36, 1968

7. Fox JL: Intracranial Aneurysms. New York: Springer-Verlag, 1983

8. Friedman JA, Pichelmann MA, Piepgras DG, et al: Ischemic complications of surgery for anterior choroidal artery aneurysms. J Neurosurg 94:565-572, 2001

9. Giannotta SL, Oppenheimer JH, Levy ML, et al: Management of intraoperative rupture of aneurysm without hypotension. Neurosurgery 28:531-536, 1991

10. Giombini S, Ferraresi S, Pluchino F: Reversal of oculomotor disorders after intracranial aneurysm surgery. Acta Neurochir 112:19-24, 1991

11. Horiuchi T, Kyoshima K, Oya F, et al: Fenestrated oculomotor nerve caused by internal carotid-posterior communicating artery aneurysm: case report. Neurosurgery 40:397-399, 1997

12. Hupperts RM, Lodder J, Heuts-van Raak EP, et al: Infarcts in the anterior choroidal artery territory. Anatomical distribution, clinical syndromes, presumed pathogenesis and early outcome. Brain 117:825-834, 1994

13. Kissel JT, Burde RM, Klingele TG, et al: Pupil-sparing oculomotor palsies with internal carotid-posterior communicating artery aneurysms. Ann Neurol 13:149-154, 1983

14. Kopitnik TA Jr, Horowitz MB, Samson DS: Surgical management of intraoperative aneurysm rupture, in, Schmidek $\mathrm{HH}$ (ed): Schmidek and Sweet Operative Neurosurgical Techniques: Indications, Methods, and Results, ed 4. Philadelphia: WB Saunders, 2000 Vol. 12, pp 1275-1281

15. Leivo S, Hernesniemi J, Luukkonen M, et al: Early surgery improves the cure of aneurysm-induced oculomotor palsy. Surg Neurol 45:430-434, 1996

16. Ojemann RG, Heros RC, Crowell RM: Surgical Management of Cerebrovascular Disease, ed 2. Baltimore: Williams \& Wilkins, 1988, pp 183-191

17. Paine JT, Batjer HH, Samson D: Intraoperative ventricular puncture. Neurosurgery 22:1107-1109, 1988

18. Rhoton AL Jr: Anatomy of saccular aneurysms. Surg Neurol 14:59-66, 1980

19. Rhoton AL Jr: Aneurysms. Neurosurgery 51 (Suppl 4): $121-158,2002$

20. Rhoton AL Jr: Microsurgical anatomy of saccular aneurysms, in Wilkins RH, Rengachary SS (eds): Neurosurgery. New York, McGraw-Hill, 1985 Vol 2, pp 1330-1340

21. Rhoton AL Jr, Fujii K, Fradd B: Microsurgical anatomy of the anterior choroidal artery. Surg Neurol 12:171-187, 1979

22. Rhoton AL Jr, Saeki N, Perlmutter D, et al: Microsurgical anatomy of common aneurysm sites. Clin Neurosurg 26: 248-306, 1979

23. Samson DS, Batjer HH: Intracranial Aneurysm Surgery: Techniques. New York: Futura, 1990

24. Wiebers DO, Whisnant JP, Sundt TM Jr, et al: The significance of unruptured intracranial saccular aneurysms. J Neurosurg 66:23-29, 1987

25.Yaşargil MG: Microneurosurgery: Clinical Considerations, Surgery of the Intracranial Aneurysms and Results. New York: Thieme-Stratton, 1984, Vol II, pp 182-183

Manuscript received April 16, 2003.

Accepted in final form May 7, 2003.

Address reprint requests to: Brian A. O'Shaughnessy, M.D., Department of Neurological Surgery, Northwestern University, McGaw Medical Center, 233 East Erie Street, Suite 614, Chicago, Illinois 60611. email: branos@md.northwestern.edu. 